\title{
Can we safely continue to offer surgical treatments during the COVID-19 pandemic?
}

\author{
Alex Fowler (1) , Tom E F Abbott (D) , Rupert M Pearse (D)
}

Critical Care \& Perioperative Medicine Research Group, William Harvey Research Institute, Queen Mary University of London, London, UK

\section{Correspondence to} Professor Rupert M Pearse, Critical Care and Perioperative Medicine Research Group, Queen Mary University of London, London E1 1BB, UK: r.pearse@qmul.ac.uk

Accepted 16 November 2020 Published Online First 20 November 2020

\section{Sinked}

- http://dx.doi.org/10.1136/ bmjqs-2020-012156

\section{Check for updates}

(c) Author(s) (or their employer(s)) 2021. No commercial re-use. See rights and permissions. Published by BMJ.

To cite: Fowler A, Abbott TEF, Pearse RM. BMJ Qual Saf 2021;30:268-270.
The coronavirus pandemic has caused enormous disruption to routine healthcare in many countries, with large numbers of patients requiring hospital treatment for COVID-19. In the UK National Health Service (NHS) many routine care pathways were rapidly suspended to redeploy staff, hospital beds and equipment to handle the massive influx of patients. Surgical procedures were cancelled for all but the most urgent cases. ${ }^{2}$ In this issue of BMJ Quality and Safety, the Corona Hands Collaborative group report the results of a cohort study conducted over a 2-week period in April 2020, during the peak of the first wave of the COVID-19 pandemic in the UK. ${ }^{3}$ Data were collected for 1093 patients undergoing upper limb surgery in 74 NHS hospitals. A clinician survey captured data describing surgical cancellations, operating room capacity and infection control procedures at each hospital. The principal finding was that, in this patient population, the risk of death within 30 days after surgery was very low $(<0.1 \%)$. While 19 patients were diagnosed with COVID-19, only one patient died, and the reported postoperative complications match the expected pattern for upper limb surgery. The authors conclude that low-risk surgery can be safely delivered in the context of the pandemic.

In any observational study, an important source of bias is misclassification of the exposure or patient outcome. In this case, the identification of patients with SARS-CoV-2 infection and diagnosis of COVID-19 warrants discussion. SARS-CoV-2 is a virus, which requires a test (usually viral PCR) to identify it. Infection with SARS-CoV-2 can be symptomatic or asymptomatic. COVID-19 is the clinical disease caused by infection with SARS-CoV-2 and, in some situations, can be diagnosed without a positive SARS-CoV-2 test result. ${ }^{4}$ Due to the limited availability of SARS-CoV-2 testing early in the pandemic, the authors' definition of SARS-CoV-2 included patients with a high probability of COVID-19, in addition to patients with SARS-CoV-2 infection confirmed by viral PCR testing. Here, 15 out of 19 patients reported to have SARS-CoV-2 infection were classified based on clinical suspicion and/or chest radiography, in the absence of a positive viral PCR test. While this may raise a concern of misclassification of exposure, in the context of a widespread pandemic, clinically detectable disease is highly likely to be COVID-19 caused by SARS-CoV-2. In future studies, it may be important to differentiate between patients with clinically diagnosed COVID-19 compared with asymptomatic SARS-CoV-2 infection.

The primary outcome was mortality within 30 days after surgery. In this sample, the authors reported a very low rate of death among patients with COVID19. This contrasts with the findings of the COVIDSurg study, a large international observational study of patients with SARS-CoV-2 infection or COVID-19 disease, in which half the cohort suffered a postoperative pulmonary complication. ${ }^{5}$ The risk of mortality among patients with a pulmonary complication was as high as 1 in 3, which contrasts with the low rate of mortality reported by the Corona Hands Collaborative. This likely relates to the broad range of procedures included in the COVIDSurg cohort, with higher baseline risk of morbidity compared with low-risk surgical procedures such as upper limb surgery. Three-quarters of patients in COVIDSurg received general anaesthesia compared with one-third in the Corona Hands study. Some commentators have 
raised concern about delivering general anaesthesia to patients with COVID-19, given the theoretically harmful impact of ventilating diseased lungs. ${ }^{6}$ This aligns with the outputs of the logistic regression model reported in the Corona Hands study, where general anaesthesia was associated with increased odds of suffering a complication. However, this should be cautiously interpreted given the low incidence of complications and potential confounding effect of patient or disease factors putting those needing general anaesthesia at greater risk of complications.

To facilitate the COVID-19 response during the first wave, the majority of elective care was suspended. Consistent with guidance from NHS England, almost all of the 400000 elective NHS surgical procedures that occur every month were cancelled. ${ }^{27}$ The Corona Hands Collaborative investigated the scale of cancellations of upper limb surgery using hospital-level survey and found that $99.7 \%$ of elective procedures were cancelled. This is consistent with our own research findings that estimate as many as 1 million NHS procedures were delayed or cancelled during the initial peak of COVID-19, with a further 1 million cancellations expected due to difficulties in delivering surgical services in the context of the pandemic. ${ }^{8}$ However, there is still considerable uncertainty about the impact of subsequent waves of COVID-19 on elective care, not limited to surgical services. A large proportion of delayed procedures are typically performed among patients who, by virtue of age or comorbidity, are at high risk of complications should they contract COVID-19. ${ }^{9}$

Protecting patients susceptible to critical illness with SARS-CoV-2 infection is paramount to ensure their well-being and to enable the ongoing provision of healthcare nationally. In wider society, measures such as social distancing and use of face coverings have been implemented to reduce prevalence and therefore risk of infection. ${ }^{10}$ In hospitals we now have enhanced infection control procedures, including routine preoperative SARS-CoV-2 testing and stringent isolation precautions. These measures have reduced surgical throughput substantially, even before we consider the additional impact from redeployment of surgical staff to care for patients with COVID-19. However, delays in care caused by infection control procedures must be balanced against the risk of bringing infected patients or staff into contact with vulnerable patients in lowrisk 'green zones' deemed to be free of COVID-19. The balance of risk and benefit for these patients is challenging and the provision of surgical care cannot continue like it did before the pandemic started. It is inevitable that surgical waiting lists will get longer with associated impact on outcomes for patients requiring time-critical treatments. There has also been a substantial reduction in secondary care referrals, including referrals into cancer pathways. ${ }^{11} 12$ This suggests that the burden of untreated surgical disease in the community is even greater than current waiting lists would indicate. While there have been many direct deaths due to COVID-19, we expect a substantial number of additional deaths due to delays in treatment among patients with other life-threatening diseases. ${ }^{13}$ At least in the UK, there is now a clear national policy intent to continue routine healthcare alongside treatment for patients with COVID-19. The prevalence of COVID-19 dipped during summer months, but the recent resurgence will inevitably result in a higher in-hospital prevalence. With regional fluctuations in community prevalence, decisions about screening, isolation and cancellation of surgery must be made dynamically. From the point of view of patients and their surgeons, this will mean regular changes in infection control policies which we know will lead to frustration. We will never completely escape the tension between the need to resume surgical treatment of diseases and the risk to individual patients who may experience worse outcomes if they contract COVID-19.

The Corona Hands Collaborative group are to be congratulated for completing this large study under exceptionally difficult circumstances. They provide reassuring data for young, fit patients who require low-risk ambulatory surgery. However, the skewed risk of severe complications associated with COVID-19 as age increases means that these findings may not be so easily applied to older patients undergoing major surgery, especially abdominal cancer surgery. ${ }^{9}$ The risks of admitting patients to hospital without rigorous infection control measures have not been explored in this study. Whatever decisions are made, the balance between harm from the disease COVID-19 and the harm caused by disruption of usual healthcare will have consequences for millions of people. Inevitably this means the decision will be made by politicians elected to represent the views of society, with advice rather than instruction from healthcare experts. Finally, one lingering question remains - what will the outcome be for the millions of patients who may never get their surgical treatment? Many patients will experience substantial harm because of delayed or cancelled operations, but not necessarily all. There has been a steady growth in surgical treatments over the last 20 years. ${ }^{714}$ While this may be expected with an ageing population, it is likely there was some growth in procedures that offer minimal patient benefit. As we reflect on the impact of the COVID-19 pandemic over the coming months and years, will we decide that some surgeries are not as necessary as we previously thought?

Twitter Tom E F Abbott @_tomabbott and Rupert M Pearse @rupert_pearse

Contributors All authors are responsible for manuscript preparation, critical editing and approval of the final submitted version. 
Funding The authors have not declared a specific grant for this research from any funding agency in the public, commercial or not-for-profit sectors.

Competing interests RMP has received honoraria and/ or research grants from the National Institute for Health Research, Edwards Lifesciences, Intersurgical and GlaxoSmithKline. AF holds a National Institute for Health Research Doctoral Research Fellowship.

Patient consent for publication Not required.

Provenance and peer review Commissioned; externally peer reviewed.

This article is made freely available for use in accordance with BMJ's website terms and conditions for the duration of the covid-19 pandemic or until otherwise determined by BMJ. You may use, download and print the article for any lawful, noncommercial purpose (including text and data mining) provided that all copyright notices and trade marks are retained.

\section{ORCID iDs}

Alex Fowler http://orcid.org/0000-0003-4461-7777

Tom E F Abbott http://orcid.org/0000-0002-8664-3001

Rupert M Pearse http://orcid.org/0000-0002-4373-5934

\section{REFERENCES}

1 COVIDSurg Collaborative. Elective surgery cancellations due to the COVID-19 pandemic: global predictive modelling to inform surgical recovery plans. Br J Surg 2020. doi:10.1002/ bjs.11746. [Epub ahead of print: 12 May 2020].

2 NHS England. Clinical guide to surgical prioritisation during the coronavirus pandemic 2020.

3 Dean BJF, Corona Hands Collaborative. Mortality and pulmonary complications in patients undergoing upper extremity surgery at the peak of the SARS-CoV-2 pandemic in the UK: a national cohort study. BMJ Qual Saf 2021;30:283-91.
4 Huang C, Wang Y, Li X, et al. Clinical features of patients infected with 2019 novel coronavirus in Wuhan, China. Lancet 2020;395:497-506.

5 Nepogodiev D, Bhangu A, Glasbey JC, et al. Mortality and pulmonary complications in patients undergoing surgery with perioperative SARS-CoV-2 infection: an international cohort study. The Lancet 2020;396:27-38.

6 Canet J, Gallart L, Gomar C, et al. Prediction of postoperative pulmonary complications in a population-based surgical cohort. Anesthesiology 2010;113:1338-50.

7 Abbott TEF, Fowler AJ, Dobbs TD, et al. Frequency of surgical treatment and related Hospital procedures in the UK: a national ecological study using Hospital episode statistics. $\mathrm{Br} \mathrm{J}$ Anaesth 2017;119:249-57.

8 Fowler AJ, Dobbs TD, Wan YI, et al. Resource requirements for reintroducing elective surgery in England during the COVID-19 pandemic: a modelling study. Br J Surg 2020.

9 Fowler AJ, Abbott TEF, Prowle J, et al. Age of patients undergoing surgery. Br J Surg 2019;106:1012-8.

10 Greenhalgh T, Schmid MB, Czypionka T, et al. Face masks for the public during the covid-19 crisis. BMJ 2020;369:m1435.

11 Sud A, Jones ME, Broggio J, et al. Collateral damage: the impact on outcomes from cancer surgery of the COVID-19 pandemic. Ann Oncol 2020;31:1065-74.

12 Sud A, Torr B, Jones ME, et al. Effect of delays in the 2-weekwait cancer referral pathway during the COVID-19 pandemic on cancer survival in the UK: a modelling study. Lancet Oncol 2020;21:1035-44.

13 Maringe C, Spicer J, Morris M, et al. The impact of the COVID-19 pandemic on cancer deaths due to delays in diagnosis in England, UK: a national, population-based, modelling study. Lancet Oncol 2020;21:1023-34.

14 Weiser TG, Haynes AB, Molina G, et al. Estimate of the global volume of surgery in 2012: an assessment supporting improved health outcomes. Lancet 2015;385 Suppl 2:S11. 